\title{
Cholinergic receptors play a role in the cardioprotective effects of anesthetic preconditioning: Roles of nitric oxide and the CaMKK//AMPK pathway
}

\author{
YANG YANG $^{1 *}$, YING LI $^{2 *}$, JIE WANG $^{1}$, LEI HONG $^{3}$, SHIGANG QIAO $^{3,4}$, CHEN WANG $^{3,4}$ and JIANZHONG AN ${ }^{3}$ \\ ${ }^{1}$ Department of Anesthesiology, Wujiang Hospital Affiliated to Nantong University, Suzhou, Jiangsu 215200; \\ ${ }^{2}$ Department of Cardiology, ${ }^{3}$ Institute of Clinical Medicine Research and ${ }^{4}$ Department of Anesthesiology and \\ Perioperative Medicine, The Affiliated Suzhou Science and Technology Town Hospital of Nanjing Medical University, \\ Suzhou, Jiangsu 215153, P.R. China
}

Received March 27, 2020; Accepted November 13, 2020

DOI: $10.3892 /$ etm.2020.9569

\begin{abstract}
Vagus nerve activation may have important therapeutic significance for myocardial ischemia-reperfusion (IR) injury. Nitric oxide (NO) plays a vital role in the cardioprotective effects of anesthetic preconditioning (APC). Moreover, acetylcholine $(\mathrm{ACh})$ prevents cardiomyocyte damage by activating AMP-activated protein kinase (AMPK) and increasing the phosphorylation of $\mathrm{Ca}^{2+} / \mathrm{calmodulin}$-dependent protein kinase $\beta$ (CaMKK $\beta)$. The aim of the present study was to determine whether APC could protect heart function by antagonizing IR damage via the cholinergic system. It was hypothesized that the NO synthase (NOS)/CaMKK $\beta / \mathrm{AMPK}$ pathway might be involved in the cardioprotective effects induced by cholinergic receptor activation. Isolated rat hearts were subjected to ischemia for $30 \mathrm{~min}$ followed by $120 \mathrm{~min}$ of reperfusion. Volatile anesthetic sevoflurane (3.5\%) was administered for $15 \mathrm{~min}$ before ischemia, then rinsed for $15 \mathrm{~min}$. The muscarinic acetylcholine receptor (mAChR) antagonist atropine (ATR; $100 \mathrm{nM}$ ) and the nicotinic acetylcholine receptor (nAChR) antagonist hexamethonium (HEM; $50 \mu \mathrm{M}$ ) were administered 10 min before APC. Both $\mathrm{mAChR}$ and $\mathrm{nAChR}$ were involved in APC-induced cardioprotection. ATR and HEM treatment both abolished the protective effects of APC on IR damage in isolated hearts, demonstrating the importance of cholinergic receptors in the protection
\end{abstract}

Correspondence to: Professor Jianzhong An, Institute of Clinical Medicine Research, The Affiliated Suzhou Science \& Technology Town Hospital of Nanjing Medical University, 1 Lijiang Road, Gaoxin, Suzhou, Jiangsu 215153, P.R. China

E-mail: szkjcyy_ajz@126.com

*Contributed equally

Key words: anesthetic preconditioning, cholinergic receptors, heart, ischemia, nitric oxide, calcium/calmodulin-dependent protein kinase kinase- $\beta$, AMPK mechanism of APC. The present study thus suggests that APC plays a cardioprotective role, in part, by regulating neurohumoral pathways. In addition, there may be functional coupling between the two cholinergic receptors, and the NOS and CaMKK $\beta$ /AMPK pathways may play roles in shared pathways that mediate the cardioprotective effects of APC. These findings may provide insight into potential new mechanisms of APC-induced cardioprotection against IR injury.

\section{Introduction}

Anesthetic preconditioning (APC) refers to exposure of the heart to a volatile anesthetic followed by its washout $(1,2)$. We previously demonstrated that APC protects the heart against subsequent ischemia-reperfusion (IR) injury $(1,2)$. Although APC is an effective strategy to reduce myocardial injury, its exact underlying mechanism remains poorly understood.

The autonomic balance between the sympathetic and parasympathetic nervous systems plays an important role in the regulation of the cardiovascular system (3). Acetylcholine $(\mathrm{ACh})$ is the main neurotransmitter of the vagus nerve. It may mimic the effects of myocardial ischemic preconditioning (IPC) and protect the heart against myocardial IR injury (4). This indicates that the activation of ACh receptors is involved in cardioprotective signaling pathways (4-6). There are two main types of cholinergic receptors in the heart, namely, the muscarinic (mAChR) and the nicotinic receptors (nAChR) $(4,7,8)$. Evidence has shown that through pharmacological or direct-current electrical stimulation, both mAChRs and nAChRs can trigger signaling pathways that protect the heart against IR injury $(9,10)$.

Nitric oxide (NO) is an endogenous regulatory molecule involved in various physiological processes (11). The endogenous NO synthase (eNOS) pathway is expressed in isolated cardiomyocytes and regulates the negative chronotropic effects of cholinergic receptor stimulation (12). In addition, activation of the neuronal NOS (nNOS)/AMPactivated protein kinase (AMPK)/mTOR pathway has been linked to the cardioprotective effects caused by IPC (13). ACh prevents cardiomyocyte damage by activating AMPK 
and inhibiting reactive oxygen species (ROS) formation (14). Recently, it was reported that during myocardial ischemia, vagus nerve stimulation (VNS) may activate AMPK, leading to the phosphorylation of calcium/calmodulin-dependent protein kinase $\beta(\mathrm{CaMKK} \beta)$ (15). This suggested that the CaMKK $\beta /$ AMPK signaling pathway is involved in VNS-mediated protective effects. However, whether and how APC regulates cholinergic receptors to prevent IR injury and improve cardiac function remains unclear. ACh initiates downstream signaling by activating G-protein-coupled $\mathrm{mAChRs}$ or by binding to nAChRs, which are ligand-gated ion channels (16). Complex neural processing occurs within the heart, not only in response to central efferent input, but also to sensory afferent information from the myocardium (17). Nevertheless, whether APC exerts cardioprotection through the upregulation of cholinergic receptors in the isolated heart remains to be determined. Therefore, the aim of the present study was to determine the role of cholinergic receptors in APC-induced cardioprotection against IR injury in an isolated rat heart model. In addition, whether the NOS and CaMKK $\beta$ /AMPK pathways are involved in the beneficial effects of cholinergic receptor activation was also examined.

\section{Materials and methods}

Animals. The present study was approved by The Institutional Animal Care and Use Committee of the Affiliated Suzhou Science \& Technology Town Hospital of Nanjing Medical University. Male, 8-10-week-old Sprague-Dawley rats $(250 \pm 50 \mathrm{~g})$ were purchased from the Animal Center of Suzhou University and housed under a 12 -h light/dark cycle at $25^{\circ} \mathrm{C}$ and $60 \%$ humidity. All rats were provided with food and water ad libitum. All animals were treated in accordance with the National Institutes of Health's Guidelines for the Care and Use of Experimental Animals.

Isolated heart preparation. Preparation of isolated heart was performed as described previously $(2,18)$. Briefly, rats were intraperitoneally anesthetized with $50 \mathrm{mg} / \mathrm{kg}$ pentobarbital sodium, then decapitated when they did not respond to a noxious stimulus to the hind limb. The heart was excised and perfused using the Langendorff method at a perfusion pressure of $80 \mathrm{mmHg}$. A thermostatically controlled water circulator (Lauda E100; Lauda) was used to maintain the temperature of the perfusion and bath at $37.2 \pm 0.1^{\circ} \mathrm{C}$. Left ventricular pressure (LVP) was measured using a volume equalizer of a saline-filled latex balloon connected to the left atrium through a mitral valve into the left ventricle. The heart was immersed in aerated physiological buffer solution at $37.2^{\circ} \mathrm{C}$ for $30 \mathrm{~min}$ of global ischemia and then reperfused for $120 \mathrm{~min}$. At the end of the experiment, the heart was frozen and kept at $-80^{\circ} \mathrm{C}$ until use.

Experimental protocols. The present experimental protocols were similar to our previous study (2). Each experiment lasted $220 \mathrm{~min}$ and a total of 60 rats were used. After $30 \mathrm{~min}$ of perfusion, when functional parameters reached equilibrium (steady state); the hearts were randomly divided into five groups ( $n=12$ hearts in each group): i) Untreated sham group: Continuous perfusion for $190 \mathrm{~min}$ without ischemia or drug administration; ii) IR group, after an additional $40 \mathrm{~min}$ of perfusion, hearts received $30 \mathrm{~min}$ of global ischemia and 120 min of reperfusion; iii) APC group, 3.5\% sevoflurane (Abbott Pharmaceutical Co. Ltd.) was administered for $15 \mathrm{~min}$, then washed out for $15 \mathrm{~min}$ priorto ischemia; iv) an atropine (ATR; mAChR antagonist; $100 \mathrm{nM}$; Sigma-Aldrich; Merck KGaA) group, used based on its affinity to $\mathrm{mAChR}$ $\left(\mathrm{K}_{\mathrm{D}}=0.36 \mathrm{nM}\right)$ and administered 10 min before APC and $\left.\mathrm{v}\right)$ an hexamethonium (HEM; nAChR antagonist; Sigma-Aldrich; Merck KGaA) group, $50 \mu \mathrm{M}$ was used to achieve specificity at nAChRs within the cardiac ganglia.

Sevoflurane was bubbled into the perfusate using an agent-specific vaporizer placed in the $\mathrm{O}_{2}-\mathrm{CO}_{2}$ gas mixture line. Samples of coronary perfusate were collected from a port in the aortic cannula to measure sevoflurane concentration by gas chromatography. Inflow sevoflurane concentration was $0.64 \pm 0.02 \mathrm{mM}$, which is equivalent to $3.34 \pm 0.22 \%$ atmosphere and a minimal alveolar concentration of $1.5 \pm 0.4 \%$ (1). The ATR and HEM groups were used to evaluate the effects of cholinergic receptors. ATR and HEM doses were selected according to a previous study (17).

Measurement of hemodynamic function. Hemodynamic parameters were monitored throughout the experiment. After $30 \mathrm{~min}$ of ischemia, hemodynamic function was assessed by determining the left ventricular developed pressure (LVDP), left ventricular end-diastolic pressure (LVEDP) and the maximal and minimal derivatives of LVP $(+\mathrm{dP} / \mathrm{dt}$ and $-\mathrm{dP} / \mathrm{dt})$. These parameters represent the major indices of myocardial contractility and relaxation. In particular, LVEDP is not only a marker of diastolic function but also a good predictor of cardiac mortality.

Myocardial high-energy phosphate analysis. At the end of reperfusion, the heart was frozen using aluminum forceps pre-cooled in liquid nitrogen to measure myocardial ATP and creatine phosphate (CP) levels, as described previously (19). Cardiac CP is converted to ATP by the enzymatic reaction of creatine kinase (20). Briefly, frozen ventricles were ground and mixed with $0.3 \mathrm{M} \mathrm{HClO}_{4}$ and $0.25 \mathrm{mM}$ EDTA under liquid nitrogen cooling. The extract was centrifuged at $8,000 \mathrm{x} g$ for $15 \mathrm{~min}$ at $4^{\circ} \mathrm{C}$, and the resulting supernatant was sampled using high-pressure liquid chromatography to measure myocardial ATP and CP.

Determination of NOS activity and NO levels. At the end of the experiment, the heart was removed and homogenized in a $0.9 \%$ ice-cold saline solution, then centrifuged at $600 \mathrm{x} \mathrm{g}$ for 10 min at $4^{\circ} \mathrm{C}$. NOS activity and NO levels were measured using a diagnostic assay kit (Nanjing Jiancheng Bioengineering Institute). The absorbance at a wavelength of $530 \mathrm{~nm}$ was measured using a DU-640 spectrophotometer (Beckman Coulter, Inc.), and normalized to the control according to the manufacturer's instructions (11).

Western blot analysis. Western blot analysis was performed as described previously (2). Hearts were homogenized using RIPA lysis buffer (cat. no. 20-188; EMD Millipore) and a complete mammalian proteinase inhibitor cocktail (cat. no. PI101; Roche Diagnostics $\mathrm{GmbH}$ ) and then 

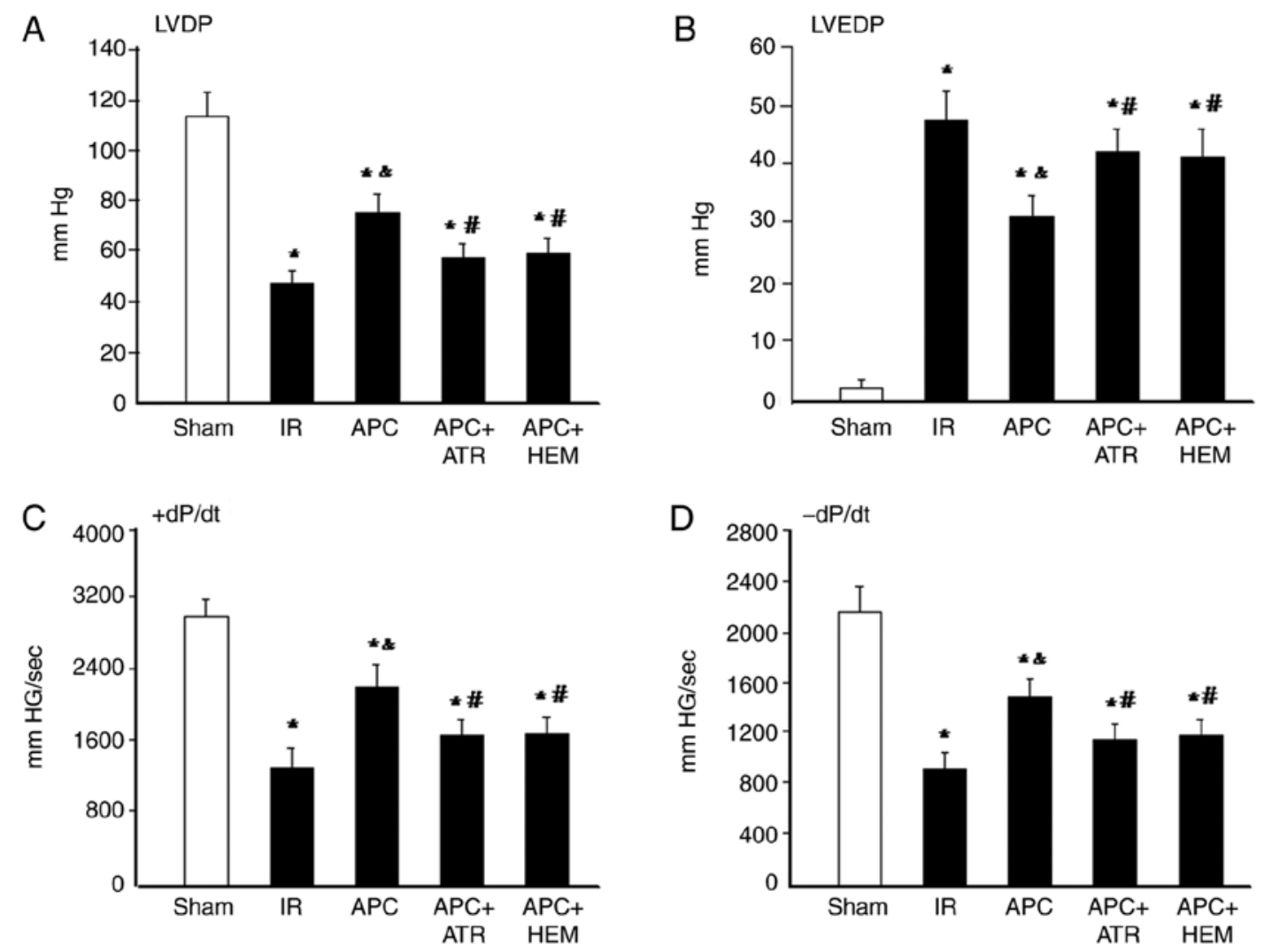

Figure 1. LVDP, LVEDP, +dP/dt and -dP/dt after IR. (A) LVDP, (B) LVEDP, (C) +dP/dt and (D) -dP/dt after 30 min ischemia and 120 min reperfusion. Values are presented as the mean \pm standard deviation. ${ }^{*} \mathrm{P}<0.05$ vs. Sham group; ${ }^{\star} \mathrm{P}<0.05$ vs. IR group; ${ }^{\mathrm{P}}<0.05$ vs. APC group. LVDP, left ventricular developed pressure; LVEDP, left ventricular end-diastolic pressure; $\pm \mathrm{dP} / \mathrm{dt}$, the maximal and minimal derivatives of LVDP; IR, ischemia-reperfusion; APC, anesthetic preconditioning; ATR, atropine; HEM, hexamethonium.

centrifuged at $13,200 \times \mathrm{g}$ for $20 \mathrm{~min}$ at $4^{\circ} \mathrm{C}$. A BCA assay kit (cat. no. P0010; Beyotime Institute of Biotechnology) was used to determine the protein concentration. After denaturation, $20 \mu \mathrm{g}$ of each sample was dissolved in Laemmli sample buffer (cat. no. S3401; Sigma-Aldrich; Merck KGaA) and separated using SDS-PAGE on a $10 \%$ gel. The samples were then transferred to a nitrocellulose membrane, which was blocked with 5\% skim milk in PBS for $1 \mathrm{~h}$ at room temperature, The membranes were subsequently incubated with primary antibody at $4^{\circ} \mathrm{C}$ overnight, and then incubated with HRP-conjugated goat anti-rabbit secondary antibody (1:10,000; cat. no. ab6721; Abcam) or HRP-conjugated goat anti-mouse secondary antibody (1:10,000; cat. no. sc-2031; Santa Cruz Biotechnology, Inc.) at room temperature for $1 \mathrm{~h}$. Protein bands were visualized using a SuperSignal West Pico kit (cat. no. 34577; Pierce; Thermo Fisher Scientific, Inc.). Band density was quantified using UN-SCAN-IT software (v.7.0; Silk Scientific, Inc.). The primary antibodies were specific for eNOS (cat. no. sc-376751; 1:1,000; Santa Cruz Biotechnology, Inc.), nNOS (cat. no. sc-5302; 1:1,000; Santa Cruz Biotechnology, Inc.); AMPK (cat. no. 2352; 1:1,000; Cell Signaling Technology, Inc.); phosphorylated (p)-AMPK (Thr172; cat. no. 5884; 1:1,000; Cell signaling Technology, Inc.); p-CaMKK2 (Ser511; cat. no. AF4487; 1:1,000; Affinity Biosciences) and CAMKK2 (polyclonal antibody; cat. no. DF4793; 1:1,000; Affinity Biosciences); as well as the housekeeping protein GAPDH (cat. no. AG019; 1:1,000; Shanghai Biyuntian Biotechnology Co., Ltd.).
Statistical analysis. Data are presented as the mean \pm SD. Each experiment was repeated at least three times. SPSS 19.0 (IBM Corp.) was used to conduct statistical analyses. One-way ANOVA was used to compare the differences among five groups, followed by Tukey's post-hoc test to determine the differences between groups. $P<0.05$ was considered to indicate a statistically significant difference.

\section{Results}

Cardiac performance. The LVDP significantly decreased following IR compared with the sham group (Fig. 1A). Moreover, the LVDP significantly increased in the APC group compared with the IR group. By contrast, APC-induced improvement of LVDP was significantly inhibited by ATR or HEM treatment $(\mathrm{P}<0.05)$. Hearts subjected to IR displayed a significant increase in LVDP (Fig. 1B). However, a significant reduction in LVEDP was observed after APC treatment. The effect of APC treatment was inhibited by ATR or HEM treatment. Cardiac contractility $(+\mathrm{dP} / \mathrm{dt})$ and relaxation $(-\mathrm{dP} / \mathrm{dt})$ (Fig. 1C and D) were reduced during ischemia in all groups. Following reperfusion, contractility increased but still remained lower than that recorded before ischemia throughout reperfusion in each group. APC significantly improved contractile recovery in IR hearts, which was also abolished by ATR or HEM treatment.

ATP and CP content. At the end of reperfusion, the ATP and $\mathrm{CP}$ content were determined in trans-mural sections obtained 

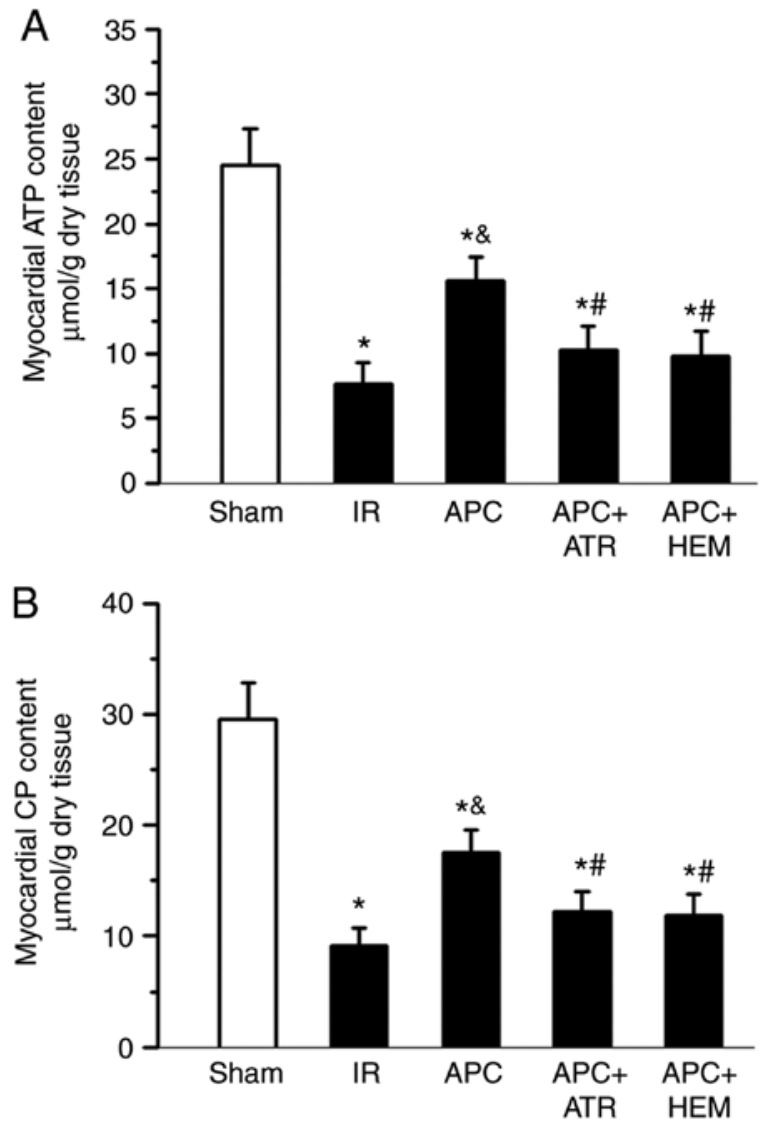

Figure 2. ATP and CP content of transmural sections obtained from the left ventricular free wall of rat hearts. (A) Myocardial ATP and (B) CP content. Values are presented as the mean $\pm \mathrm{SD}$. ${ }^{*} \mathrm{P}<0.05$ vs. Sham group; ${ }^{\circledR} \mathrm{P}<0.05$ vs. IR group. ${ }^{~} \mathrm{P}<0.05$ vs. APC group. $\mathrm{CP}$, creatine phosphate; IR, ischemia-reperfusion; APC, anesthetic preconditioning; ATR, atropine; HEM, hexamethonium.

from the left ventricular free wall (Fig. 2). In the IR groups, the ATP content was significantly reduced $(7.7 \pm 1.6 \mu \mathrm{mol} / \mathrm{g})$ compared with the sham group $(24.5 \pm 2.8 \mu \mathrm{mol} / \mathrm{g}$; Fig. $2 \mathrm{~A})$. In addition, the $\mathrm{CP}$ content was also significantly decreased in the IR group compared with the sham group (9.2 $\pm 1.5 \mathrm{vs}$. $29.6 \pm 3.3 \mu \mathrm{mol} / \mathrm{g}$; Fig. 2B). However, the levels of ATP and $\mathrm{CP}$ were better preserved in the APC-treated group (15.6 \pm 1.8 and $17.5 \pm 2.1 \mu \mathrm{mol} / \mathrm{g}$ ) compared with the IR group. Both the mAChR antagonist ATR and $n A C h R$ antagonist HEM abolished the preserving effects of APC.

Effects of APC on NOS activity and NO content. Following IR, both NOS activity and NO levels were significantly decreased in the IR group compared with the sham group (Fig. 3). Moreover, NOS activity and NO levels significantly increased in the APC group compared with the IR group (Fig. 3A and B). However, ATR and HEM treatment abolished the APC-induced increase in NOS activity and NO levels. These results indicated that both NOS activity and NO levels were decreased following IR injury, but increased after APC treatment. However, the effects of APC were reversed by ATR and HEM administration.

Effects of APC on eNOS and nNOS phosphorylation. Western blotting bands of eNOS, p-eNOS, nNOS and phosphorylated nNOS (p-nNOS) protein in Fig. 4A. The percentile ratio of
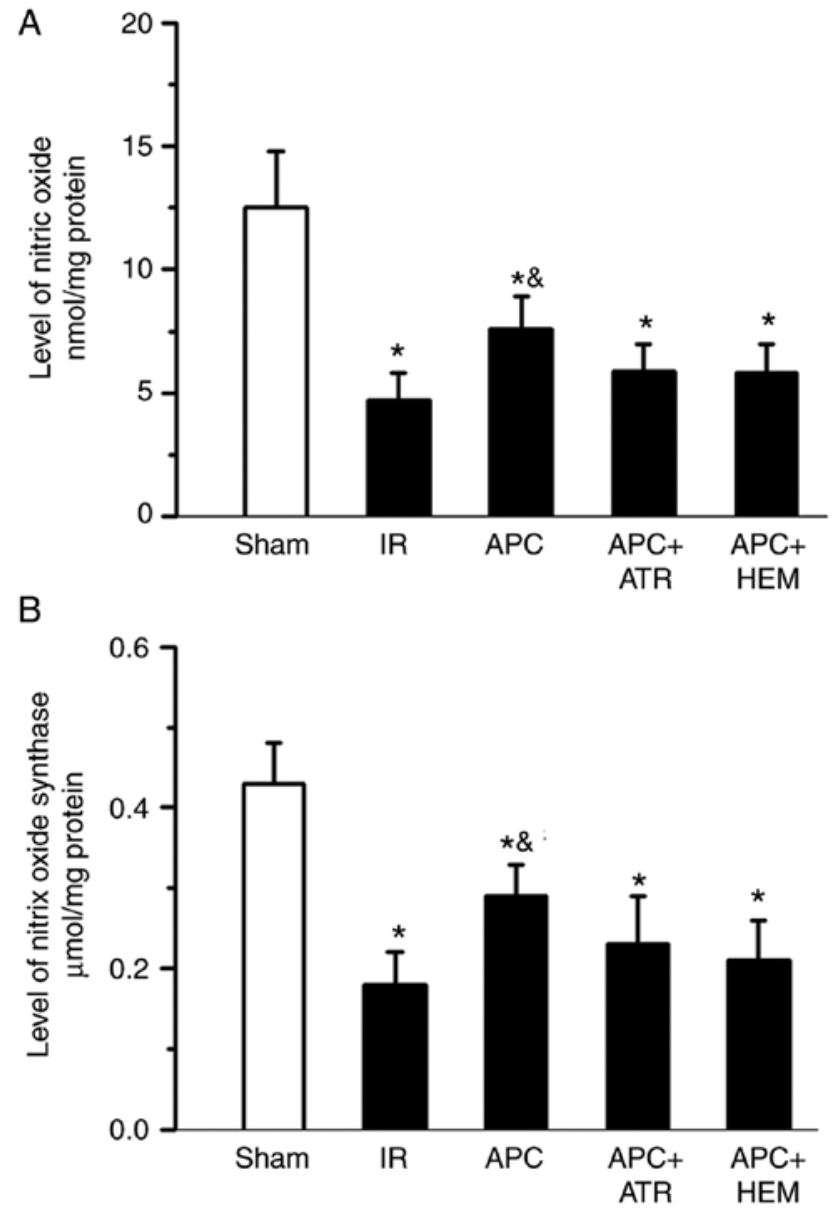

Figure 3. APC increases the levels of (A) NO and (B) NOS activity. APC-induced increase of NO levels and NOS activity was inhibited by the muscarinic acetylcholine receptor antagonist, ATR $(100 \mathrm{nM})$ and the nicotinic acetylcholine receptor antagonist $\operatorname{HEM}(50 \mu \mathrm{M})$. The data are presented as the mean \pm SD. $n=5$ hearts/group. ${ }^{*} \mathrm{P}<0.05$ vs. Sham group; ${ }^{\&} \mathrm{P}<0.05$ vs. IR group. NO, nitric oxide; NOS, NO synthase, IR, ischemia-reperfusion; APC, anesthetic preconditioning; ATR, atropine; HEM, hexamethonium.

p-eNOS/total eNOS and p-nNOS/total nNOS are displayed in Fig. 4B and C. There was a significant increase in eNOS (Fig. 4B) and nNOS (Fig. 4C) phosphorylation in the IR group compared with the sham group. Treatment with APC further increased the phosphorylation of eNOS and nNOS (APC group vs. IR group). However, this effect was significantly attenuated following ATR and HEM administration (APC+ATR group or APC+HEM group vs. APC group).

Effects of SPC on AMPK and CaMKK $\beta$ phosphorylation. Western blotting bands of AMPK and p-AMPK(Thr172) protein and the ratio of $\mathrm{p}-\mathrm{AMPK}(\mathrm{Thr172}) /$ total AMPK are presented in Fig. 5A. Western blotting bands of CAMKK $\beta$ and $\mathrm{p}-\mathrm{CAMKK} \beta$ (Ser511) protein and the ratio of $\mathrm{p}-\mathrm{CAMKK} \beta$ (Ser511)/total CAMKK $\beta$ are displayed in Fig. 5B. There was a significant decrease in AMPK (Fig. 5A) and CAMKK $\beta$ (Fig. 5B) phosphorylation in the IR group compared with the sham group. Treatment with APC significantly increased the phosphorylation of AMPK and CAMKK $\beta$ (APC group vs. IR group). Following ATR and HEM administration, this effect was significantly reduced (APC+ATR group or APC+HEM group, vs. APC group). 

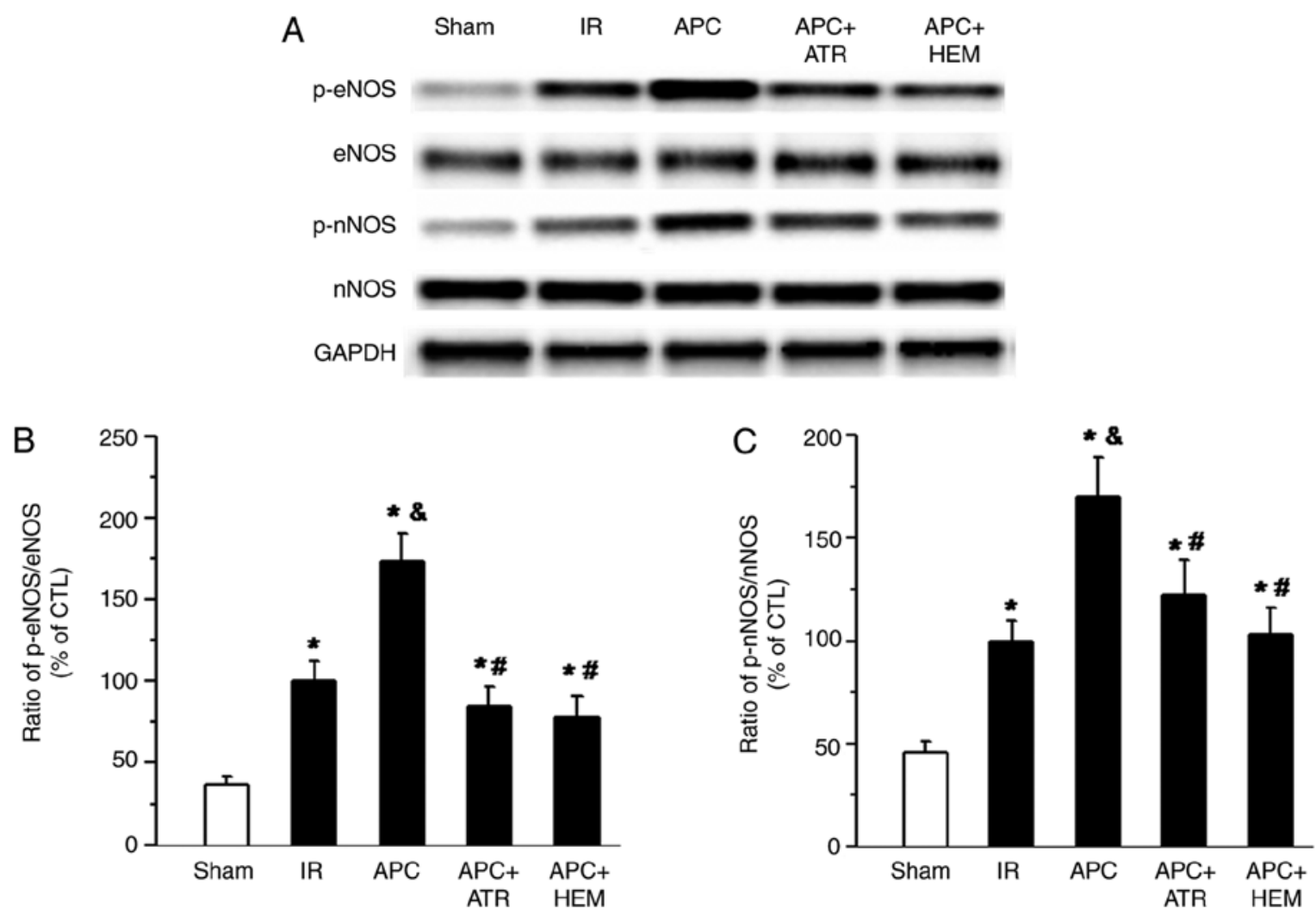

Figure 4. Western blot analysis of eNOS, nNOS, p-eNOS and p-nNOS levels in the homogenates of myocardial tissue from rat hearts. (A) Western blot bands of eNOS, p-eNOS, nNOS and p-nNOS. The ratio of (B) p-eNOS/total eNOS and (C) p-nNOS/total nNOS. APC-induced increases of the phosphorylation of eNOS and nNOS were reduced by the muscarinic acetylcholine receptor antagonist ATR $(100 \mathrm{nM})$ and nicotinic acetylcholine receptor antagonist HEM $(50 \mu \mathrm{M})$. The data are presented as the mean \pm SD. $\mathrm{n}=5$ hearts/group. " $\mathrm{P}<0.05$ vs. Sham group; ${ }^{\mathrm{P}} \mathrm{P}<0.05$ vs. IR group; $\mathrm{P}<0.05$ vs. APC group. eNOS, endogenous nitric oxide synthase; nNOS, neuronal NOS; p, phosphorylated; IR, ischemia-reperfusion; APC, anesthetic preconditioning; ATR, atropine; HEM, hexamethonium; CTL, control.

\section{Discussion}

Our recent study demonstrated that APC reduced myocardial enzyme release and infarct size by enhancing the recovery of cardiac function, thereby reducing myocardial damage after IR (2). Possible mechanisms underlying the role of cholinergic receptors in alleviating IR injury have been proposed in several previous studies, including NOS and ROS-mediated CaMKII pathways $(14,15,21,22)$. However, whether and how APC regulates the intrinsic cardiac nervous system to improve cardiac function remains unknown. The current study demonstrated that both mAChRs and nAChRs participate in APC-induced cardioprotection in isolated rat hearts following IR. ATR and HEM attenuated the protective effects of APC against IR injury, highlighting the importance of cholinergic receptors in the mechanism of APC-induced cardioprotection. Thus, the present findings indicated that APC plays a cardioprotective role, in part, by regulating neurohumoral pathways. In addition, NOS and CaMKK $\beta / A M P K$ may be involved in shared pathways that mediate the cardioprotective mechanisms of APC.

Previous studies suggested that increased vagal nerve activity could reduce myocardial IR injury $(3,4,7,23)$. The main vagal neurotransmitter $\mathrm{ACh}$ can replicate the cardioprotective effects of IPC $(17,24,25)$. Upon pharmacological or direct-current stimulation, both $\mathrm{mAChR}$ and $\mathrm{nAChR}$ can trigger cardioprotective signaling cascades, which are effective against I/R injury (21). In addition, previous studies have demonstrated that cardiomyocytes synthesized and secreted $\mathrm{ACh}$, which provided further evidence for the importance of non-neurocholinergic signaling cascades in the maintenance of myocardial function in physiological and pathological states (26-28). A recent study examined the role of the intrinsic cardiac nervous system in the classic myocardial IPC mechanism and demonstrated that intrinsic cardiac ganglia remain intact in isolated hearts subjected to IR injury (17). In addition, IPC activated the intrinsic cardiac nerve reflex, leading to the release of $\mathrm{ACh}$ in the ventricle and inducing protective effects through the activation of cholinergic receptors. Treatment with ATR and HEM also blocked the protective effects of IPC (17). The present study was consistent with these previous findings. APC reduced IR injury by activating intrinsic cardiac cholinergic receptors, and this protective effect was abrogated by ATR and HEM, indicating that APC protected the heart against IR injury via intrinsic neuronal mechanisms.

NO plays a number of beneficial roles during myocardial reperfusion, including regulating myocardial contractility, opening $\mathrm{K}_{\text {ATP }}$ channels to the sarcolemma and mitochondria, antioxidant effects and oxygen free radical production $(29,30)$. Our previous study indicated that NO played a vital role in the cardioprotective effects of APC (11). APC alleviated cardiac dysfunction caused by IR, reduced the area of infarction after ischemia, and led to higher levels of eNOS and nNOS 
A
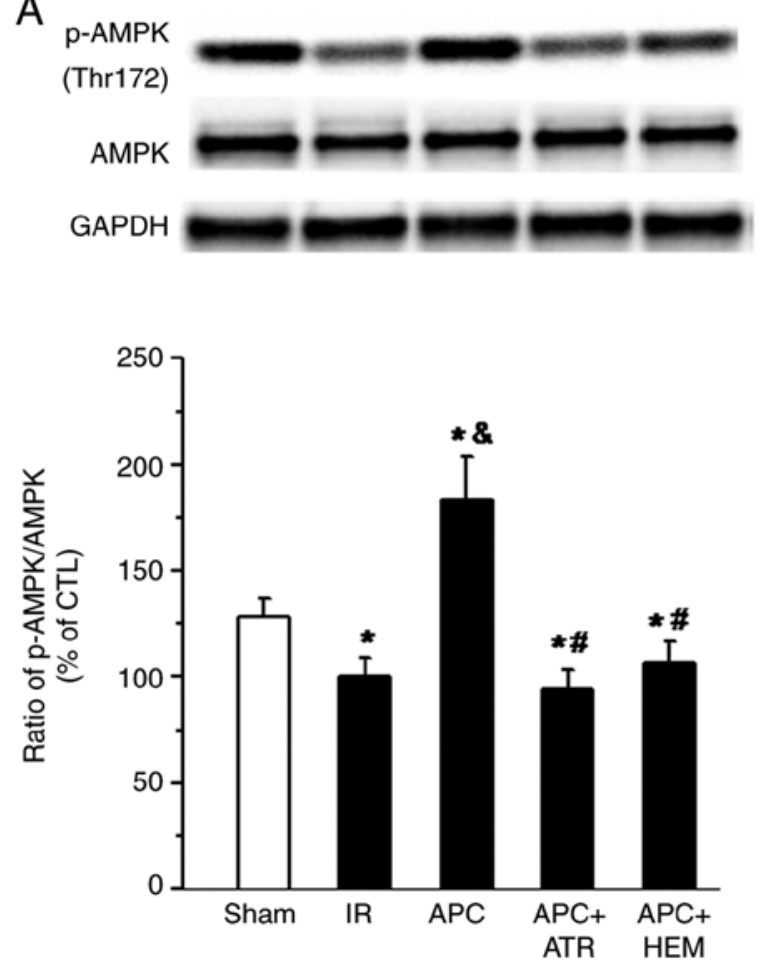

B p-CAMKK $\beta$

(Ser511)

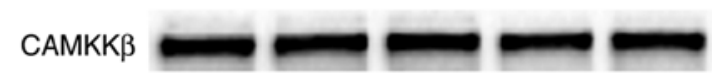

GAPDH

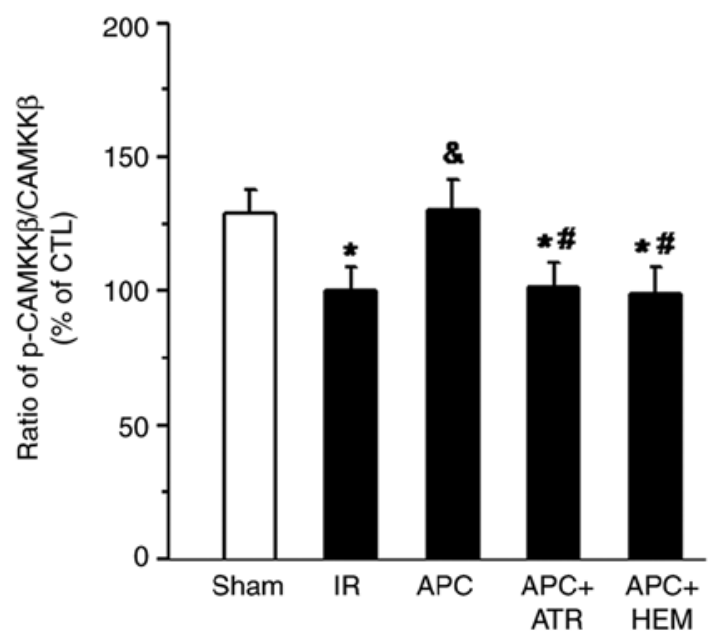

Figure 5. Western blot analysis of AMPK, CAMKK $\beta$, AMPK phosphorylation at Thr-172 and CAMKK $\beta$ phosphorylation at Ser-511 in the homogenates of myocardial tissue from rat hearts. (A) Western blot bands of AMPK and p-AMPK(Thr172) protein and the ratio of p-AMPK(Thr172)/total AMPK. (B) Western blot bands of CAMKK $\beta$ and p-CAMKK $\beta($ Ser511) protein and the ratio of p-CAMKK $\beta($ Ser511)/total CAMKK $\beta$. APC-induced increases of AMPK and CAMKK $\beta$ phosphorylation were reduced by the muscarinic acetylcholine receptor antagonist atropine (ATR, $100 \mathrm{nM}$ ) and nicotinic acetylcholine receptor antagonist hexamethonium (HEM, $50 \mu \mathrm{M})$. The data are presented as the mean \pm standard deviation. $\mathrm{n}=5$ hearts/group. ${ }^{*} \mathrm{P}<0.05$ vs. Sham group; ${ }^{\&} \mathrm{P}<0.05$ vs. IR group; ${ }^{\#} \mathrm{P}<0.05$ vs. APC group. CAMKK $\beta$, calcium/calmodulin-dependent protein kinase kinase $\beta$; p, phosphorylated; IR, ischemia-reperfusion; APC, anesthetic preconditioning; ATR, atropine; HEM, hexamethonium; CTL, control.

phosphorylation, NOS content and NO production. The present study is consistent with our previous reports $(31,32)$. NO, a neurosensor of parasympathetic nerves, had a significant effect on the promotion of vagus nerves by increasing the release of $\mathrm{ACh}$ and reducing the downstream effects of catecholamines on heart rate and contractility (33). Thus, control of cardiac contraction through eNOS activation may represent an important function of cholinergic receptor activation (34). In the present study, the mAChR antagonist ATR and the $\mathrm{nAChR}$ antagonist HEM eliminated the effects of APC on eNOS and nNOS phosphorylation, increased NOS content and NO production and antagonized the protective effects of APC on the heart. These results indicated that eNOS and nNOS phosphorylation is one of the downstream pathways of APC-induced cholinergic receptor activation and cardioprotection.

AMPK is a key cellular energy sensor and regulator of metabolic homeostasis (15). Increasing evidence suggested that AMPK dysfunction is associated with the occurrence and development of a number of cardiovascular diseases, including atherosclerosis, myocardial IR injury and cardiac remodeling (35-37). Activation of AMPK protected the myocardium from IR injury by regulating mitochondrial function (38) and preventing myocardial necrosis and systolic dysfunction (39). Other studies reported that VNS activates AMPK and is accompanied by CaMKK $\beta$ phosphorylation during myocardial ischemia (15). These findings suggested that the intrinsic cardiac nervous system may be involved in APC-mediated cardioprotective effects against IR injury through the cholinergic receptor and CaMKK $\beta / A M P K$ signaling during myocardial IR. The present study also demonstrated that APC significantly increased the phosphorylation of AMPK and CaMKK $\beta$, and that the administration of ATR and HEM could suppress this increase in phosphorylation. This indicated that the activation of the CaMKK $\beta / A M P K$ signaling pathway by cholinergic receptors was involved in APC-mediated cardioprotection. Taken together, these results represented an important complement to the understanding of the role of cholinergic receptors in APC-induced cardioprotection against IR injury. Nonetheless, the myocardial protective properties of volatile anesthetics, including sevoflurane, may also be due to their cardiosuppressive nature. These cardiac depressant effects decrease myocardial oxygen demand and may thus improve the myocardial oxygen balance during ischemia (40). A previous study demonstrated that both isoflurane and sevoflurane increased coronary blood flow and decreased coronary vascular resistance, including resistance through the collateral circulation (41). Therefore, further research is required to ascertain the effects of APC on the microcirculation under myocardium ischemia conditions.

The present study has its limitations. Although HEM is widely used as a nAChR antagonist, it also has low affinity to $\mathrm{M}_{2}$ receptors (17). Therefore, in the present study, $50 \mu \mathrm{M}$ was used in order to achieve high specificity to nicotinic receptors. Nevertheless, the potential nonspecific effects of HEM still remains a possibility. Moreover, the interaction between NO 
and the CaMKK $\beta /$ AMPK signaling pathway is still unclear. In addition, it was reported that cholinergic receptor activation also plays a cardioprotective role through other pathways, such as, phosphoinositide 3-kinase and Erk1/2 signaling (42), Bcl-2 family proteins and caspase-3-related pathways (42), as well as Akt and GSK-3 $\beta$ enzyme activity (43). The interaction between these signal pathways is still poorly understand. Lastly, cholinergic receptor activation has previously been reported to mediate apoptosis and oxidative stress during I/R-induced cell injury (21). However, how apoptosis, oxidative stress and CaMKK $\beta / \mathrm{AMPK}$ pathways regulate cholinergic receptors to resist IR injury through APC was not evaluated in the present study.

In summary, the present study demonstrated that APC could protect cardiac function against IR injury through the activation of cholinergic receptor-mediated eNOS, nNOS and CaMKK $\beta /$ AMPK phosphorylation. The present findings may provide insight into novel mechanisms of APC-induced cardioprotection against IR injury.

\section{Acknowledgements}

The authors acknowledge Dr Tuanjie Che (Precision Medicine and Translational Medicine Laboratory, Suzhou Science \& Technology Town Hospital, China) for his technical assistance in the study.

\section{Funding}

The present study was supported by the Suzhou Science and Technology Development Plan (grants nos. SS201756 and SS201613); the Suzhou New District Science and Technology Project (grant no. 2017Z004) and the National Science and Technology Development Plan (grant no. NSFC 81703501).

\section{Availability of data and materials}

The datasets used and/or analyzed during the current study are available from the corresponding author on reasonable request.

\section{Authors' contributions}

YY, YL, CW and JA were responsible for experimental design, data collection, data analysis and manuscript writing. YY, YL, JW, LH and SQ performed the experiments. JA revised manuscript critically for important intellectual content. All authors read and approved the final manuscript.

\section{Ethics approval and consent to participate}

The present study was approved by The Institutional Animal Care and Use Committee of the Affiliated Suzhou Science \& Technology Town Hospital of Nanjing Medical University (grant no. IRB2018032).

\section{Patient consent for publication}

Not applicable.

\section{Competing interests}

The authors declare that they have no competing interests.

\section{References}

1. An J, Varadarajan SG, Novalija E and Stowe DF: Ischemic and anesthetic preconditioning reduces cytosolic $[\mathrm{Ca} 2+]$ and improves $\mathrm{Ca}(2+)$ responses in intact hearts. Am J Physiol Heart Circ Physiol 281: H1508-H1523, 2001.

2. Wang C, Qiao S, Hong L, Sun J, Che T, An J and Camara AKS: NOS cofactor tetrahydrobiopterin contributes to anesthetic preconditioning induced myocardial protection in the isolated ex vivo rat heart. Int J Mol Med 45: 615-622, 2020

3. Zhang J, Yong Y, Li X, Hu Y, Wang J, Wang YQ, Song W, Chen WT, Xie J, Chen XM, et al: Vagal modulation of high mobility group box-1 protein mediates electroacupunctureinduced cardioprotection in ischemia-reperfusion injury. Sci Rep 5: 15503, 2015.

4. Nuntaphum W, Pongkan W, Wong jaikam S, Thummasorn S, Tanajak P, Khamseekaew J, Intachai K, Chattipakorn SC, Chattipakorn $\mathrm{N}$ and Shinlapawittayatorn K: Vagus nerve stimulation exerts cardioprotection against myocardial ischemia/reperfusion injury predominantly through its efferent vagal fibers. Basic Res Cardiol 113: 22, 2018.

5. Zhao M, He X, Bi XY, Yu XJ, Gil Wier W and Zang WJ: Vagal stimulation triggers peripheral vascular protection through the cholinergic anti-inflammatory pathway in a rat model of myocardial ischemia/reperfusion. Basic Res Cardiol 108: 345, 2013.

6. Wang Z, Yu L, Wang S, Huang B, Liao K, Saren G, Tan T and Jiang $\mathrm{H}$ : Chronic intermittent low-level transcutaneous electrical stimulation of auricular branch of vagus nerve improves left ventricular remodeling in conscious dogs with healed myocardial infarction. Circ Heart Fail 7: 1014-1021, 2014.

7. Uitterdijk A, Yetgin T, te Lintel Hekkert M, Sneep S, Krabbendam-Peters I, van Beusekom HM, Fischer TM, Cornelussen RN, Manintveld OC, Merkus D and Duncker DJ: Vagal nerve stimulation started just prior to reperfusion limits infarct size and no-reflow. Basic Res Cardiol 110: 508, 2015.

8. Zhang R, Wugeti N, Sun J, Yan H, Guo Y, Zhang L, Ma M, Guo X, Jiao C, Xu W, et al: Effects of vagus nerve stimulation via cholinergic anti-inflammatory pathway activation on myocardial ischemia/reperfusion injury in canine. Int J Clin Exp Med 7: 2615-2623, 2014.

9. Calvillo L, Vanoli E, Andreoli E, Besana A, Omodeo E, Gnecchi M, Zerbi P, Vago G, Busca G and Schwartz PJ: Vagal stimulation, through its nicotinic action, limits infarct size and the inflammatory response to myocardial ischemia and reperfusion. J Cardiovasc Pharmacol 58: 500-507, 2011.

10. Kiss A, Tratsiakovich Y, Mahdi A, Yang J, Gonon AT, Podesser BK and Pernow J: Vagal nerve stimulation reduces infarct size via a mechanism involving the -7 nicotinic acetylcholine receptor and downregulation of cardiac and vascular arginase. Acta Physiol 221: 174-181, 2017.

11. Qiao SG, Sun Y, Sun B, Wang A, Qiu J, Hong L, An JZ, Wang C and Zhang HL: Sevoflurane postconditioning protects against myocardial ischemia/reperfusion injury by restoring autophagic flux via an NO-dependent mechanism. Acta Pharmacol Sin 40: 35-45, 2019.

12. Balligand JL, Kobzik L, Han X, Kaye DM, Belhassen L, O'Hara DS, Kelly RA, Smith TW and Michel T: Nitric oxide-dependent parasympathetic signaling is due to activation of constitutive endothelial (type III) nitric oxide synthase in cardiac myocytes. J Biol Chem 270: 14582-14586, 1995.

13. Hao M, Zhu S, Hu L, Zhu H, Wu X and Li Q: Myocardial ischemic postconditioning promotes autophagy against ischemia reperfusion injury via the activation of the nNOS/AMPK/mTOR pathway. Int J Mol Sci 18: 614, 2017.

14. Bi X, He X, Xu M, Zhao M, Yu X, Lu X and Zang W: Acetylcholine ameliorates endoplasmic reticulum stress in endothelial cells after hypoxia/reoxygenation via M3 AChR-AMPK signaling. Cell Cycle 14: 2461-2472, 2015.

15. Xue RQ, Sun L, Yu XJ, Li DL and Zang WJ: Vagal nerve stimulation improves mitochondrial dynamics via an M3 receptor/CaMKKbeta/AMPK pathway in isoproterenol-induced myocardial ischaemia. J Cell Mol Med 21: 58-71, 2017. 
16. Mavropoulos SA, Khan NS, Levy ACJ, Faliks BT, Sison CP, Pavlov VA, Zhang Y and Ojamaa K: Nicotinic acetylcholine receptor-mediated protection of the rat heart exposed to ischemia reperfusion. Mol Med 23: 120-133, 2017.

17. Pickard JMJ, Burke N, Davidson SM and Yellon DM: Intrinsic cardiac ganglia and acetylcholine are important in the mechanism of ischaemic preconditioning. Basic Res Cardiol 112: 11, 2017.

18. An J, Rhodes SS, Jiang MT, Bosnjak ZJ, Tian M and Stowe DF: Anesthetic preconditioning enhances $\mathrm{Ca}^{2+}$ handling and mechanical and metabolic function elicited by $\mathrm{Na}^{+} / \mathrm{Ca}^{2+}$ exchange inhibition in isolated hearts. Anesthesiology 105: 541-549, 2006.

19. Fang L, Xu Z, Lu J, Hong L, Qiao S, Liu L and An J: Cardioprotective effects of triiodothyronine supplementation against ischemia reperfusion injury by preserving calcium cycling proteins in isolated rat hearts. Exp Ther Med 18: 4935-4941, 2019.

20. Sasamori J, Abe Y, Marunouchi T, Manome Y, Uchibori T and Tanonaka K: Effects of 2-octynyladenosine (YT-146) on mitochondrial function in ischemic/reperfused rat hearts. Biol Pharm Bull 38: 1946-1953, 2015.

21. Intachai K, C Chattipakorn S, Chattipakorn $\mathrm{N}$ and Shinlapawittayatorn K: Revisiting the cardioprotective effects of acetylcholine receptor activation against myocardial ischemia/reperfusion injury. Int J Mol Sci 19: 2466, 2018.

22. Zhao M, Sun L, Yu XJ, Miao Y, Liu JJ, Wang H, Ren J and Zang WJ: Acetylcholine mediates AMPK-dependent autophagic cytoprotection in $\mathrm{H} 9 \mathrm{c} 2$ cells during hypoxia/reoxygenation injury. Cell Physiol Biochem 32: 601-613, 2013.

23. Shinlapawittayatorn K, Chinda K, Palee S, Surinkaew S, Kumfu S, Kumphune S, Chattipakorn S, KenKnight BH and Chattipakorn N: Vagus nerve stimulation initiated late during ischemia, but not reperfusion, exerts cardioprotection via amelioration of cardiac mitochondrial dysfunction. Heart Rhythm 11: 2278-2287, 2014.

24. Palee S, Apaijai N, Shinlapawittayatorn K, Chattipakorn SC and Chattipakorn N: Acetylcholine attenuates hydrogen peroxide-induced intracellular calcium dyshomeostasis through both muscarinic and nicotinic receptors in cardiomyocytes. Cell Physiol Biochem 39: 341-349, 2016.

25. Li DL, Liu BH, Sun L, Zhao M, He X, Yu XJ and Zang WJ: Alterations of muscarinic acetylcholine receptors-2, 4 and $\alpha 7$-nicotinic acetylcholine receptor expression after ischaemia/reperfusion in the rat isolated heart. Clin Exp Pharmacol Physiol 37: 1114-1119, 2010.

26. Roy A,Fields WC, Rocha-Resende C, Resende RR, Guatimosim S, Prado VF, Gros R and Prado MA: Cardiomyocyte-secreted acetylcholine is required for maintenance of homeostasis in the heart. FASEB J 27: 5072-5082, 2013.

27. Kakinuma Y, Akiyama T, Okazaki K, Arikawa M, Noguchi T and Sato T: A non-neuronal cardiac cholinergic system plays a protective role in myocardium salvage during ischemic insults. PLoS One 7: e50761, 2012.

28. Oikawa S, Kai Y, Tsuda M, Ohata H, Mano A, Mizoguchi N, Sugama S, Nemoto T, Suzuki K, Kurabayashi A, et al: Non-neuronal cardiac cholinergic system influences CNS via the vagus nerve to acquire a stress-refractory propensity. Clin Sci (Lond) 130: 1913-1928, 2016.

29. Pang L, Cai Y, Tang EH, Yan D, Kosuru R, Li H, Irwin MG, $\mathrm{Ma} \mathrm{H}$ and $\mathrm{Xia} \mathrm{Z}$ : Cox-2 inhibition protects agains hypoxia/reoxygenation-induced cardiomyocyte apoptosis via Akt-dependent enhancement of iNOS expression. Oxid Med Cell Longev 2016: 3453059, 2016.
30. Li XD, Yang YJ, Geng YJ, Zhao JL, Zhang HT, Cheng YT and Wu YL: Phosphorylation of endothelial NOS contributes to simvastatin protection against myocardial no-reflow and infarction in reperfused swine hearts: Partially via the PKA signaling pathway. Acta Pharmacol Sin 33: 879-887, 2012.

31. Cao J, Xie H, Sun Y, Zhu J, Ying M, Qiao S, Shao Q, Wu H and Wang C: Sevoflurane post-conditioning reduces rat myocardial ischemia reperfusion injury through an increase in NOS and a decrease in phopshorylated NHE1 levels. Int J Mol Med 36: $1529-1537,2015$.

32. Ge ZD, Pravdic D, Bienengraeber M, Pratt PF Jr, Auchampach JA, Gross GJ, Kersten JR and Warltier DC: Isoflurane postconditioning protects against reperfusion injury by preventing mitochondrial permeability transition by an endothelial nitric oxide synthase-dependent mechanism. Anesthesiology 112: 73-85, 2010.

33. Sun L, Lu J, Yu XJ, Li DL, Xu XL, Wang B, Ren KY, Liu JK and Zang WJ: Adenine sulfate improves cardiac function and the cardiac cholinergic system after myocardial infarction in rats. J Pharmacol Sci 115: 205-213, 2011.

34. Waid DK, Chell M and El-Fakahany EE: M(2) and M(4) muscarinic receptor subtypes couple to activation of endothelial nitric oxide synthase. Pharmacology 61: 37-42, 2000.

35. Dong Y, Zhang M, Liang B, Xie Z, Zhao Z, Asfa S, Choi HC and Zou MH: Reduction of AMP-activated protein kinase alpha2 increases endoplasmic reticulum stress and atherosclerosis in vivo. Circulation 121: 792-803, 2010.

36. Kataoka Y, Shibata R, Ohashi K, Kambara T, Enomoto T, Uemura Y, Ogura Y, Yuasa D, Matsuo K, Nagata T, et al: Omentin prevents myocardial ischemic injury through AMP-activated protein kinase- and Akt-dependent mechanisms. J Am Coll Cardiol 63: 2722-2733, 2014

37. Viollet B, Horman S, Leclerc J, Lantier L, Foretz M, Billaud M, Giri S and Andreelli F: AMPK inhibition in health and disease. Crit Rev Biochem Mol Biol 45: 276-295, 2010.

38. Mukherjee D, Ghosh AK, Bandyopadhyay A, Basu A, Datta S, Pattari SK, Reiter RJ and Bandyopadhyay D: Melatonin protects against isoproterenol-induced alterations in cardiac mitochondrial energy-metabolizing enzymes, apoptotic proteins, and assists in complete recovery from myocardial injury in rats. J Pineal Res 53: 166-179, 2012.

39. Groenendyk J, Sreenivasaiah PK, Kim DH, Agellon LB and Michalak M: Biology of endoplasmic reticulum stress in the heart. Circ Res 107: 1185-1197, 2010

40. Lin E and Symons JA: Volatile anaesthetic myocardial protection: A review of the current literature. HSR Proc Intensive Care Cardiovasc Anesth 2: 105-109, 2010.

41. Turek Z, Sykora R, Matejovic M and Cerny V: Anesthesia and the microcirculation. Semin Cardiothorac Vasc Anesth 13: 249-258, 2009

42. Harvey KL, Hussain A and Maddock HL: Ipratropium bromide-mediated myocardial injury in in vitro models of myocardial ischaemia/reperfusion. Toxicol Sci 138: 457-467, 2014

43. Buchholz B, Donato M, Perez V, Deutsch ACR, Höcht C, Del Mauro JS, Rodríguez M and Gelpi RJ: Changes in the loading conditions induced by vagal stimulation modify the myocardial infarct size through sympathetic-parasympathetic interactions. Pflugers Arch 467: 1509-1522, 2015 